\title{
Exclusive and diffractive quarkonium-pair production at the LHC and FCC
}

\author{
V. P. Gonçalves $\odot$ and R. Palota da Silva \\ High and Medium Energy Group, Instituto de Física e Matemática, Universidade Federal de Pelotas, \\ Caixa Postal 354, 96010-900 Pelotas, RS, Brazil
}

(Received 6 December 2019; accepted 10 February 2020; published 21 February 2020)

\begin{abstract}
The production of a quarkonium pair in exclusive and diffractive processes in $p p$ collisions at the LHC and FCC energies is investigated. We consider the $J / \Psi J / \Psi$ and $\Upsilon \Upsilon$ production in these processes and present predictions for the transverse momentum and rapidity distributions considering the kinematical ranges expected to be covered by central and forward detectors. Results for the cross sections are also presented. Our results indicate that the double $J / \Psi$ production is dominated by the exclusive process, while the double $\Upsilon$ production receives a large contribution of the diffractive process. The impact of modeling of the gap survival factor on our predictions is discussed.
\end{abstract}

DOI: $10.1103 /$ PhysRevD.101.034025

\section{INTRODUCTION}

In the last years, the production of a quarkonium pair $(J / \Psi J / \Psi$ and $\Upsilon \Upsilon)$ has been the subject of a large number of studies [1-22]. Such analysis was strongly motivated by the experimental data from the D0 [23] Collaboration at Tevatron and the ATLAS, CMS, and LHCb Collaborations at the LHC [24-26], which have indicated that the contribution of the double partonic scattering is not negligible in inclusive proton-proton collisions, where both colliding protons dissociate in the interaction. In addition, the $\mathrm{LHCb}$ Collaboration have also released the first data for the exclusive double $J / \Psi$ production [27], where both colliding protons remain intact and the final state is characterized by two rapidity gaps, i.e., empty regions in pseudorapidity that separate the intact very forward protons from the $J / \Psi J / \Psi$ state. The study of the exclusive reactions is expected to improve our understanding about the interplay between the small- and large-distance regimes of quantum chromodynamics (QCD) [28]. As pointed out in Refs. [29,30], a quarkonium pair can also be produced in double diffractive reactions, which also are characterized by two rapidity gaps and two intact protons in the final state, but the quarkonium pair is generated by the interaction between gluons of the Pomeron (IP), which is a color-singlet object inside the proton. The results presented in Ref. [30] indicated that the contribution of the double diffractive reaction for the double $J / \Psi$ production is similar to those derived in Ref. [31] considering the exclusive

Published by the American Physical Society under the terms of the Creative Commons Attribution 4.0 International license. Further distribution of this work must maintain attribution to the author(s) and the published article's title, journal citation, and DOI. Funded by SCOAP ${ }^{3}$. production. Such result motivates the analysis to be performed in this paper, where we will present a comparison between the predictions for the double quarkonium production in diffractive and exclusive reactions. In particular, we will update the predictions derived in Ref. [30] considering a more recent parametrization for the diffractive gluon distribution, which was obtained in Ref. [32] by fitting the latest HERA data for diffractive $e p$ reactions. Moreover, we will present, for the first time, our predictions for the diffractive double quarkonium production at the energies of the Future Circular Collider (FCC) [33]. In addition, we will extend the formalism present in Ref. [31] for the double $\Upsilon$ production and estimate, for the first time, the prediction of this final state in exclusive reactions at the LHC and FCC. Our goal is to determine the kinematical range of dominance of these two reactions channels in order to be able to use future experimental data to constrain the underlying assumptions present in the description of diffractive and exclusive processes.

This paper is organized as follows. In the next section, we present a brief review of the formalism used to describe the quarkonium-pair production in central exclusive and double diffractive processes. Moreover, we discuss the treatment of the soft survival effects. In Sec. III, we present our results for the double $J / \Psi$ and $\Upsilon$ production at the LHC and FCC. Predictions for the rapidity and transverse momentum distributions are presented, and the dependence of our results on the parton distribution functions used in the calculations is discussed. Finally, in Sec. IV, our main conclusions are summarized.

\section{FORMALISM}

The typical diagrams that characterize the quarkoniumpair production in double diffractive and exclusive 

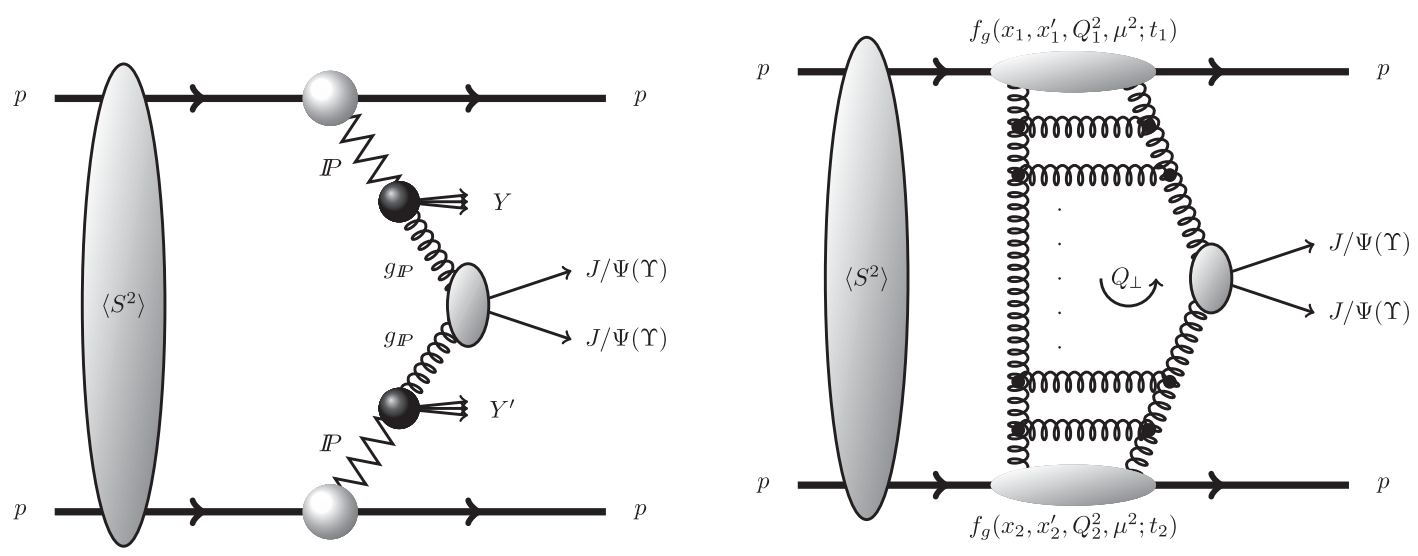

FIG. 1. Typical diagrams for the quarkonium - pair production in double diffractive (left panel) and exclusive (right panel) processes. The blob denoted by $\left\langle S^{2}\right\rangle$ represents the gap survival factor associated to absorptive effects (see text for details).

processes are represented in Fig. 1. Differently from the exclusive case, where only the quarkonium pair will be present in the final state, we have in the double diffractive process the presence of additional particles in the final state, associated to the remnants of the Pomeron, which dissociates in the interaction. The presence of these particles can be used, in principle, to discriminate the diffractive from the exclusive double quarkonium production. However, due to the large pileup of events in each bunching crossing expected to be present in the future runs of LHC and FCC, it is not clear if the separation of the diffractive and exclusive events will be possible by measuring the rapidity gaps and counting the number of tracks in the final state. As a consequence, events characterized by two rapidity gaps probably will only be separated by tagging the intact hadrons in the final state using forward detectors, as, e.g., the CMS-Totem Precision Proton Spectrometer and Atlas Forward Proton Spectrometers, associated with the ATLAS and CMS central detectors [34]. As exclusive and double diffractive events are characterized by intact hadrons, it is fundamental to know what is the relative contribution of each of these processes for the quarkonium-pair production at central and forward rapidities as well as for LHC and FCC energies. In our calculations, we will not include the double parton scattering contribution for central exclusive and diffractive processes since it is strongly suppressed due to the very small probability that two simultaneous interactions occur without the fragmentation of one (or both) of the incident protons (for a detailed discussion, see, e.g., Ref. [31]).

In what follows, we will present a brief review of the formalism proposed to describe the processes represented in Fig. 1, which is based on the resolved Pomeron model [35] in the diffractive case and in the Durham model [36] in the exclusive case. The cross section for the double diffractive cross section can be estimated using the nonrelativistic QCD (NRQCD) factorization formalism [37] for the quarkonium production and is given by [30]

$$
\begin{aligned}
& d \sigma(p p \rightarrow p+\mathcal{Q} \mathcal{Q}+p) \\
& =\sum_{n} g_{p}^{D}\left(x_{1}, \mu^{2}\right) g_{p}^{D}\left(x_{2}, \mu^{2}\right) \cdot d \hat{\sigma}\left[g g \rightarrow Q \bar{Q}_{n}+Q \bar{Q}_{n}\right] \\
& \quad \cdot\left\langle\mathcal{O}_{n}^{\mathcal{Q}}\right\rangle\left\langle\mathcal{O}_{n}^{\mathcal{Q}}\right\rangle,
\end{aligned}
$$

where $\mathcal{Q}$ represents the quarkonium state, $g^{D}$ is the diffractive gluon distribution probed at the scale $\mu^{2}$, which we assume to be equal to $\mu^{2}=M^{2}+p_{T}^{2}$, and $d \hat{\sigma}$ is the differential cross section for the $g g \rightarrow Q \bar{Q}_{n}+Q \bar{Q}_{n}$ subprocess, which is perturbatively calculated considering the production of the heavy quark pair $Q \bar{Q}$ in an intermediate Fock state $n$, which does not have to be color neutral. Moreover, the quantities $\left\langle\mathcal{O}_{n}^{\mathcal{Q}}\right\rangle$ are nonperturbative long distance matrix elements, which describe the transition of the intermediate $Q \bar{Q}$ in the physical state $\mathcal{Q}$ via soft gluon radiation and are determined from experimental data. As in Ref. [30], we will estimate $d \hat{\sigma}$ taking into account of the 31 diagrams that contribute for the color-singlet channel, as well as the 72 diagrams for the color-octet channel. Moreover, we will assume the following values for the color-octet large-distance matrix elements: $\left\langle O_{8}^{J / \psi}\left({ }^{3} S_{1}\right)\right\rangle=$ $3.9 \times 10^{-3} \mathrm{GeV}^{3}[38]$ and $\left\langle O_{8}^{\Upsilon}\left({ }^{3} S_{1}\right)\right\rangle=1.5 \times 10^{-1} \mathrm{GeV}^{3}$ [39]. In the case of the color-singlet channel, it is possible to express the matrix elements in terms of the square of the radial wave function of the quarkonium $\mathcal{Q}$ at the origin $\left(\left|R_{\mathcal{Q}}(0)\right|^{2}\right)$ which is related to the leptonic decay rate $\Gamma\left(\mathcal{Q} \rightarrow e^{+} e^{-}\right)$[40]. Therefore, such quantity can be determined, e.g., using the recent PDG data [41]. Finally, in order to calculate the double diffractive cross section, we should assume a model for the diffractive gluon distribution. As in our previous studies [29,30], we will describe this quantity using the resolved Pomeron model [35], which implies that $g_{p}^{D}\left(x, Q^{2}\right)$ is defined as a convolution of the Pomeron flux emitted by the proton, 
$f_{I P}^{p}\left(x_{I P}\right)$, and the gluon distribution in the Pomeron, $g_{I P}\left(\beta, Q^{2}\right)$, with $\beta$ being the momentum fraction carried by the partons inside the Pomeron. Such quantities can be constrained using the experimental data from diffractive deep inelastic scattering at HERA. Differently from Ref. [30], where we have used the fit B obtained by the H1 Collaboration at DESY-HERA [42] several years ago, in what follows we will consider the more recent parametrization obtained in Ref. [32] using the high-precision data from H1/ZEUS combined inclusive diffractive cross sections measurements. We will use the fit A proposed in Ref. [32], which we will denote GKG-FitA hereafter.

The central exclusive processes are usually described using the Durham model [36], proposed many years ago and extensively discussed in the literature (for a review see, e.g., Ref. [43]). In this model, the cross section for the central exclusive production of a quarkonium-pair can be expressed in terms of the skewed unintegrated gluon distributions $f_{g}$ and the subamplitude for the $g g \rightarrow \mathcal{Q Q}$ process [31]. At leading logarithmic approximation, it is possible to express $f_{g}\left(x, x^{\prime}, Q_{t}^{2}, \mu^{2}\right)$ in terms of the conventional integral gluon density $g(x)$ and the Sudakov factor $T$, which ensures that the active gluons that participate in the hard process do not radiate in the evolution from $Q_{t}$ up to the hard scale $\mu=m_{\perp} \equiv \sqrt{M_{\mathcal{Q}}^{2}+p_{\mathcal{Q}, \perp}^{2}}$. The amplitude for the $g g \rightarrow \mathcal{Q} \mathcal{Q}$ process can be estimated using the hard exclusive formalism proposed in Refs. [44,45] and considering the nonrelativistic approximation. The results presented in Ref. [31] demonstrated that the exclusive reaction is only sensitive to the color-singlet component of the meson wave function, do not receiving color-octet contributions. The final expressions for the double $J / \Psi$ production have been included in the publicly available SuperChic Monte Carlo (MC) [46]. In order to also estimate the double $\Upsilon$ production, we have modified the SuperChic and included this final state, which allows us to perform a full MC simulation of quarkonium-pair production in central exclusive processes. As in Ref. [31], we have fixed the value of the $\Upsilon$ wave function at the origin to its leptonic width. Moreover, in our calculations, we have considered that the conventional gluon distribution is given by the MMHT2014 parametrization [47]. As demonstrated in Ref. [31], the Durham predictions agree with the shape of the invariant mass distribution for the double $J / \Psi$ production in $p p$ collisions at 7 and $8 \mathrm{TeV}$ measured by the LHCb Collaboration [27]. Unfortunately, due to the limited statistics, the uncertainty in the normalization is still large.

One important open question in the description of central exclusive and diffractive interactions in $p p$ collisions is the treatment of the soft interactions that are expected to lead to extra production of particles, which will destroy the rapidity gaps in the final state and modify the associated cross sections [48]. The experimental results from Tevatron and LHC for these processes have demonstrated that these additional absorption effects cannot be neglected. Theoretically, the soft rescattering corrections associated to reinteractions (often referred to as multiple scatterings) between spectator partons of the colliding protons imply the violation of the QCD hard scattering factorization theorem for diffraction [49]. Such corrections modify the resolved Pomeron and Durham predictions for the diffractive and central exclusive processes. The modeling of the soft multiple scattering in diffractive $p p$ collisions has been the subject of several studies during the last years. For example, in Refs. [50-52], the authors have proposed to treat these effects using a general purpose Monte Carlo. However, such approaches are still strongly dependent on the treatment of the multiple interactions, the assumptions for the color flow along the rapidity gap as well as the modeling of possible proton excitations. In the case of the exclusive processes, the Durham group proposed an approach to treat the absorptive corrections associated to the additional soft proton-proton interactions (denoted eikonal factor $S_{\text {eik }}^{2}$ ), which are independent of the hard processes, as well the rescatterings of the protons with the intermediate partons that are described by the so-called

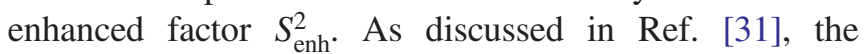
magnitude of the enhanced factor is still uncertain, but it is expected to generate a weaker suppression in comparison to that associated to the eikonal survival factor. In the case of the predictions for the double $J / \Psi$ production in exclusive processes presented in Ref. [31], the enhanced corrections were not included in the calculations. In what follows, we also will assume this approximation. In Table I, we present the predictions for the total cross sections for the quarkonium-pair production in $p p$ collisions at the LHC and FCC considering the four different models for the eikonal factor $S_{\text {eik }}^{2}$ present in the SuperChic MC, which are based on different approaches for the description of the diffractive data [53]. The main difference between these models is the distinct construction of the Good-Walker (GW) eigenstates that diagonalize the transition matrix and the data used to constrain the free parameters (for a detailed discussion, see Ref. [53]). One has that the distinct treatments of $S_{\text {eik }}^{2}$ implies that the predictions can differ by a factor $\approx 3$. It is important to emphasize that future LHC data

TABLE I. Total cross sections for the quarkonium-pair production in central exclusive processes considering $p p$ collisions at $\sqrt{s}=14 \mathrm{TeV}$ and different models for the absorptive factor. Values in parentheses are for FCC energies $(\sqrt{s}=100 \mathrm{TeV})$.

\begin{tabular}{lcc}
\hline \hline Absorptive factor & $\sigma_{2 J / \Psi}[\mathrm{pb}]$ & $\sigma_{2 \Upsilon}[\mathrm{pb}]$ \\
\hline$\left\langle S_{\text {eik }}^{2}\right\rangle$-Model 1 & $13.8(132.1)$ & $9.3 \times 10^{-5}\left(8.1 \times 10^{-4}\right)$ \\
$\left\langle S_{\text {eik }}^{2}\right\rangle$-Model 2 & $48.1(352.5)$ & $2.4 \times 10^{-4}\left(2.2 \times 10^{-3}\right)$ \\
$\left\langle S_{\text {eik }}^{2}\right\rangle$-Model 3 & $37.7(250.1)$ & $1.6 \times 10^{-4}\left(1.7 \times 10^{-3}\right)$ \\
$\left\langle S_{\text {eik }}^{2}\right\rangle$-Model 4 & $20.6(196.7)$ & $1.2 \times 10^{-4}\left(1.2 \times 10^{-3}\right)$ \\
$\left\langle S^{2}\right\rangle=0.02(0.01)$ & $39.3(439.2)$ & $1.6 \times 10^{-3}\left(2.4 \times 10^{-2}\right)$ \\
\hline \hline
\end{tabular}


for diffractive processes, in particular for the diffractive dissociation cross section, will allow to constrain the description of the GW eigenstates, and consequently, to reduce the uncertainty in $S_{\text {eik }}^{2}$ (see, e.g., [54]). Another possible approach, largely used in the literature (see, e.g., Refs. [55-64]), is based on the assumption that the hard process occurs on a short enough timescale such that the physics that generate the additional particles can be factorized and accounted by an overall factor, denoted gap survival factor $\left\langle S^{2}\right\rangle$, multiplying the cross section [65]. In general, the values of $\left\langle S^{2}\right\rangle$ depend on the energy, being typically of order 0.01-0.05 for LHC energies. In particular, for the quarkonium-pair production in double diffractive interactions in $p p$ collisions at $\sqrt{s}=14(100) \mathrm{TeV}$ it is expected to be 0.02 (0.01), i.e., the absorptive corrections are expected to suppress the cross section by a factor 50 (100). For comparison, in Table I, we also present the results derived multiplying by $\left\langle S^{2}\right\rangle$ the SuperChic predictions calculated without the inclusion of the absorptive corrections. For LHC energy and the double $J / \Psi$ production, the resulting predictions are similar to those derived with $S_{\text {eik. }}^{2}$. For FCC energy, the prediction is slightly larger. In contrast, for the double $\Upsilon$ production, we predict larger cross sections assuming the overall factor $\left\langle S^{2}\right\rangle$ instead of $S_{\text {eik }}^{2}$. As the modeling, magnitude, and universality of absorptive corrections are still a theme of intense debate $[43,66,67]$, in what follows we will assume that the absorptive corrections for the double diffractive and central exclusive processes can be modeled by the same factor $\left\langle S^{2}\right\rangle$. Surely such assumption can and must be improved in the future. However, considering the current large theoretical uncertainty in the treatment of the soft interactions, we believe that such simplistic approach allows us, at least, to understand what are the main differences between the diffractive and exclusive quarkonium-pair production associated to the distinct approaches for the hard process.

\section{RESULTS}

In what follows, we will present our predictions for the transverse momentum and rapidity distributions as well for the cross sections considering the diffractive and central exclusive quarkonium-pair production in $p p$ collisions at the LHC and FCC energies. We will present results for the typical rapidity ranges covered by central $(-2.5 \leq y \leq 2.5)$ and forward $(2.0 \leq y \leq 4.5)$ detectors. For the cross sections, we also will present our predictions for $\sqrt{s}=27 \mathrm{TeV}$,
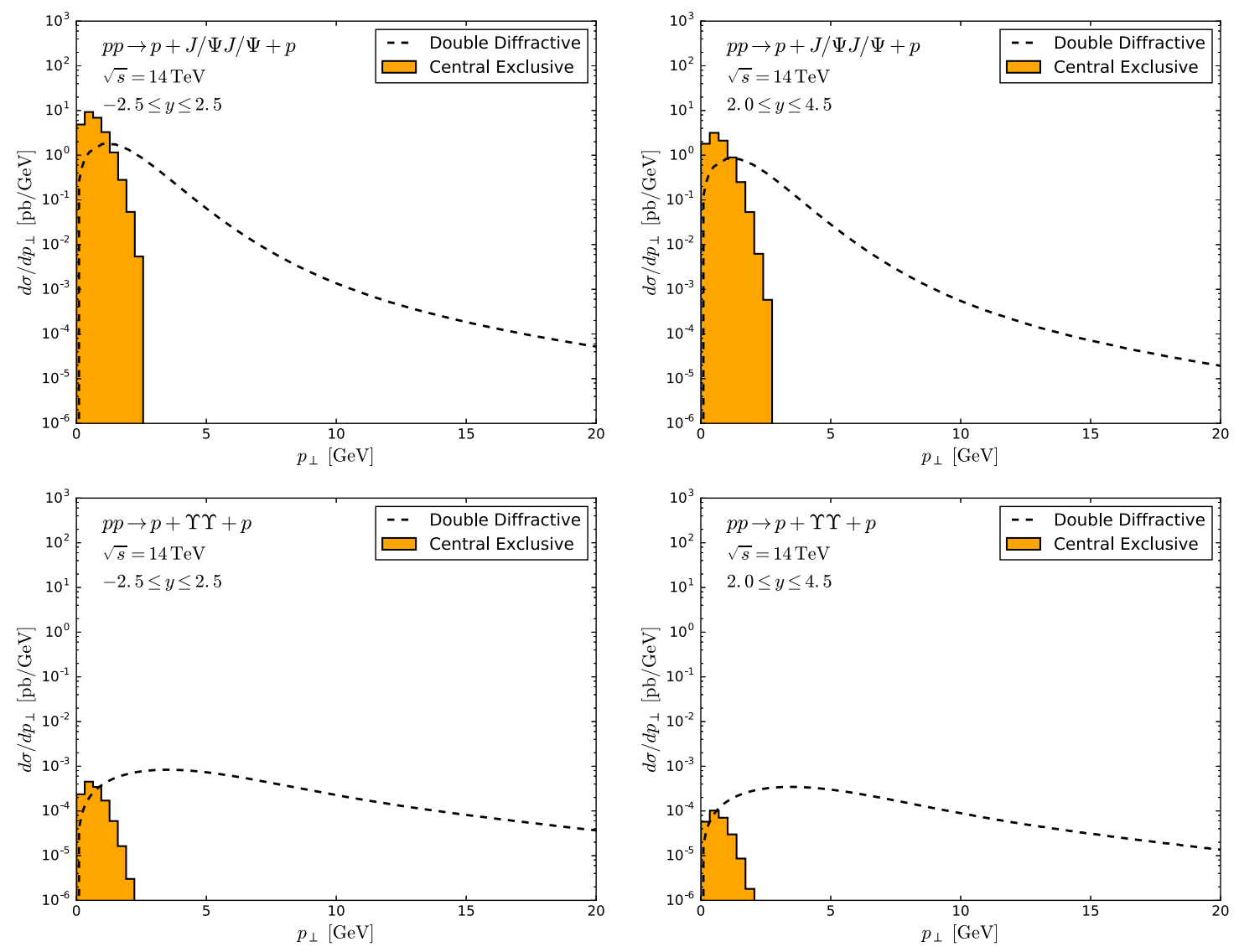

FIG. 2. Transverse momentum distributions for the diffractive and central exclusive $J / \Psi J / \Psi$ (upper panels) and $\Upsilon \Upsilon$ (lower panels) production in $p p$ collisions at $\sqrt{s}=14 \mathrm{TeV}$ considering the rapidity ranges covered by a central (left panels) and a forward (right panels) detector. 
which is the center-of-mass energy expected to be achieved in the high-energy Large Hadron Collider (HE-LHC) [68]. In our analysis, we will assume that $\left|R_{J / \Psi}(0)\right|^{2}=0.56 \mathrm{GeV}^{3}$ and $\left|R_{\Upsilon}(0)\right|^{2}=2.21 \mathrm{GeV}^{3}$. The predictions for the transverse momentum distribution are presented in Fig. 2 considering $p p$ collisions at $\sqrt{s}=14 \mathrm{TeV}$. We have verified that similar results are obtained for the energies of HE-LHC and FCC, with the main difference being the normalization of the distributions. For the double diffractive production, we have that the distribution decreases with $p_{\perp}$ following a power-law behavior $\propto 1 / p_{\perp}^{n}$, where the effective power $n$ is dependent of the final state considered. Such behavior is expected, since the quarkonium pair in the final state in diffractive interactions is generated in a $2 \rightarrow 2$ subprocess. In contrast, in the exclusive production, we have that the typical transverse momentum of the quarkonium pair is determined by the transferred momentum in the Pomeronproton vertex. As the exclusive cross section has an $e^{-\beta|t|}$
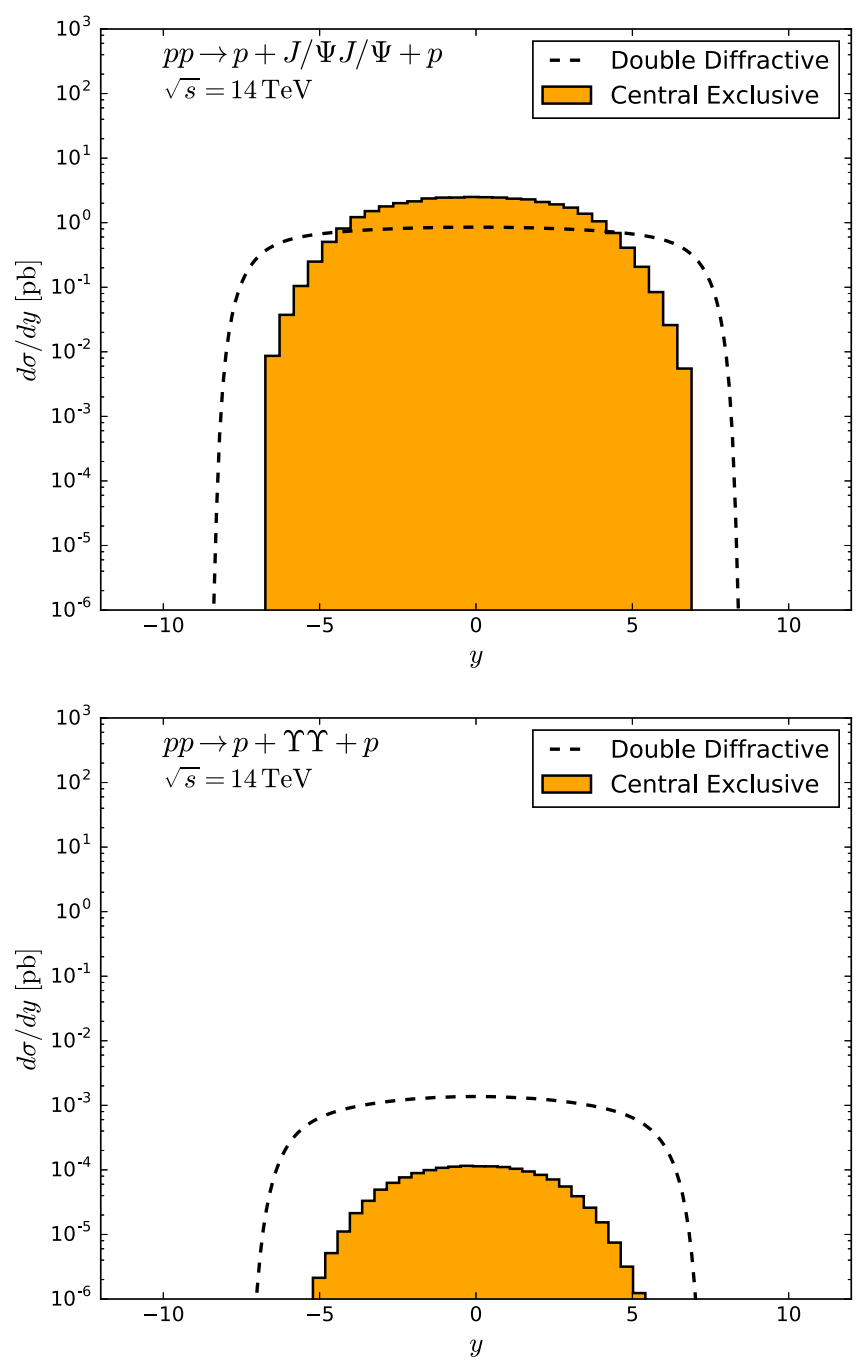

behavior, where $\beta$ is the slope parameter associated, the associated $p_{\perp}$ distribution decreases exponentially at large transverse momentum. Therefore, it is expected that the production of a quarkonium pair with a large $p_{\perp}$ should be dominated by the diffractive mechanism. On the other hand, if only events with $p_{\perp} \leq 1 \mathrm{GeV}$ are selected, the observed quarkonium pairs will be mainly produced by the exclusive process. It is important to emphasize that our results also indicate that the contribution of the diffractive process for the double $\Upsilon$ production will not be negligible at small $p_{\perp}$.

In Fig. 3, we present our predictions for the rapidity distributions considering $p p$ collisions at LHC (left panels) and FCC (right panels) energies. We have that the diffractive mechanism implies wider distributions. Moreover, our results indicate that the production of a double $J / \Psi$ at midrapidities will be dominated by the central exclusive process, with the dominance increasing with the energy. In contrast, we predict the dominance of the diffractive process in the case of double $\Upsilon$ production at the LHC.
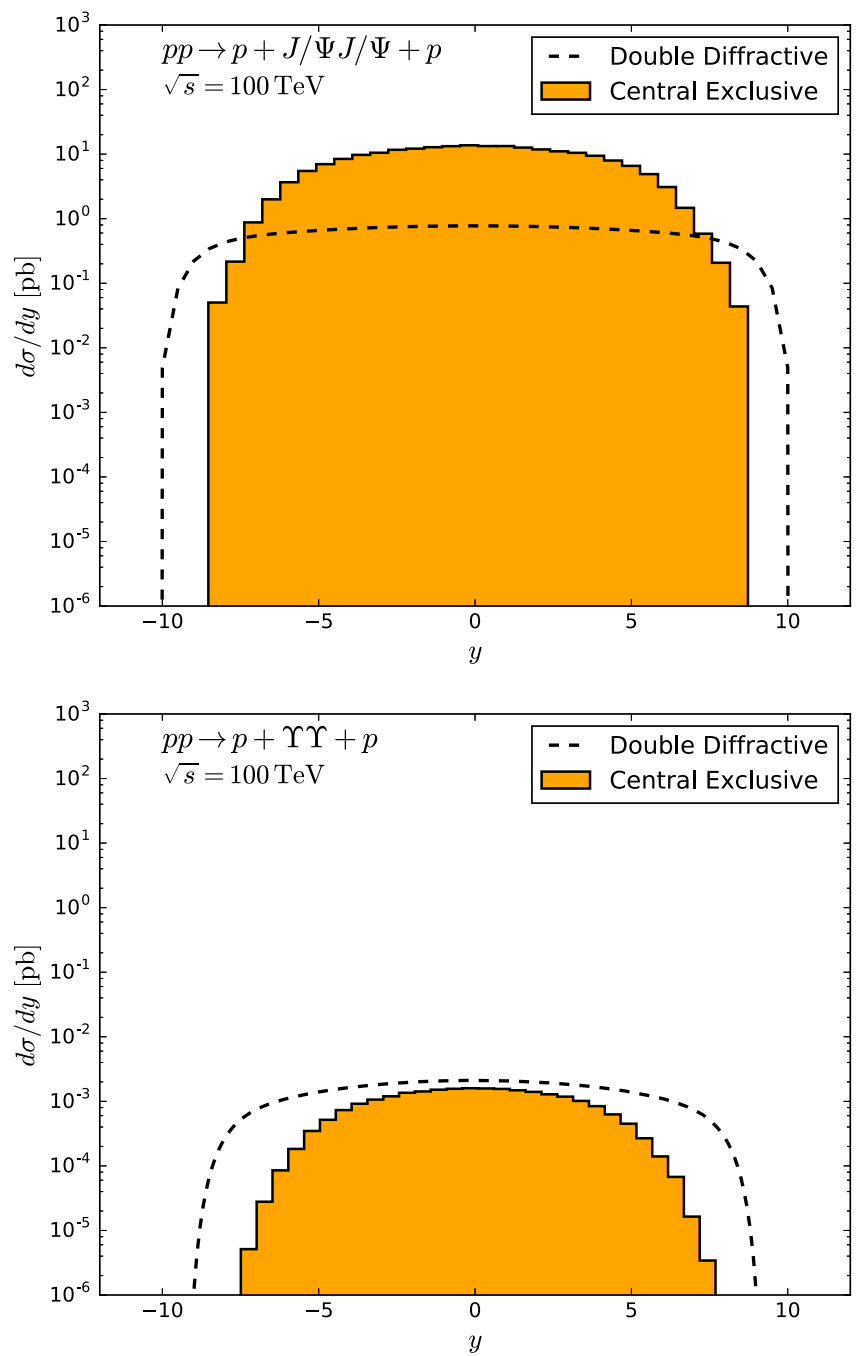

FIG. 3. Rapidity distributions for diffractive and central exclusive double $J / \Psi$ (upper panels) and double $\Upsilon$ (lower panels) production in $p p$ collisions at the LHC (left panels) and FCC (right panels) energies. 
TABLE II. Cross sections for the quarkonium-pair production in double diffractive (DD) and central exclusive processes (CEP) considering $p p$ collisions at the LHC, HE-LHC, and FCC energies.

\begin{tabular}{|c|c|c|c|c|}
\hline Energy & Process & Full rapidity range & $-2.5 \leq y \leq 2.5$ & $2.0 \leq y \leq 4.5$ \\
\hline $14 \mathrm{TeV}$ & $\begin{array}{l}\sigma_{D D}(p p \rightarrow p+J / \Psi J / \Psi+p) \\
\sigma_{C E P}(p p \rightarrow p+J / \Psi J / \Psi+p) \\
\sigma_{D D}(p p \rightarrow p+\Upsilon \Upsilon+p) \\
\sigma_{C E P}(p p \rightarrow p+\Upsilon \Upsilon+p)\end{array}$ & $\begin{array}{c}10.2 \mathrm{pb} \\
39.3 \mathrm{pb} \\
1.2 \times 10^{-2} \mathrm{pb} \\
1.6 \times 10^{-3} \mathrm{pb}\end{array}$ & $\begin{array}{c}3.7 \mathrm{pb} \\
25.9 \mathrm{pb} \\
6.5 \times 10^{-3} \mathrm{pb} \\
1.3 \times 10^{-3} \mathrm{pb}\end{array}$ & $\begin{array}{c}1.7 \mathrm{pb} \\
5.1 \mathrm{pb} \\
2.7 \times 10^{-3} \mathrm{pb} \\
2.7 \times 10^{-5} \mathrm{pb}\end{array}$ \\
\hline $27 \mathrm{TeV}$ & $\begin{array}{l}\sigma_{D D}(p p \rightarrow p+J / \Psi J / \Psi+p) \\
\sigma_{C E P}(p p \rightarrow p+J / \Psi J / \Psi+p) \\
\sigma_{D D}(p p \rightarrow p+\Upsilon \Upsilon+p) \\
\sigma_{C E P}(p p \rightarrow p+\Upsilon \Upsilon+p)\end{array}$ & $\begin{array}{c}10.3 \mathrm{pb} \\
85.0 \mathrm{pb} \\
1.5 \times 10^{-2} \mathrm{pb} \\
3.9 \times 10^{-3} \mathrm{pb}\end{array}$ & $\begin{array}{c}3.9 \mathrm{pb} \\
50.9 \mathrm{pb} \\
7.3 \times 10^{-3} \mathrm{pb} \\
2.8 \times 10^{-3} \mathrm{pb}\end{array}$ & $\begin{array}{c}1.8 \mathrm{pb} \\
18.3 \mathrm{pb} \\
3.0 \times 10^{-3} \mathrm{pb} \\
7.5 \times 10^{-4} \mathrm{pb}\end{array}$ \\
\hline $100 \mathrm{TeV}$ & $\begin{array}{l}\sigma_{D D}(p p \rightarrow p+J / \Psi J / \Psi+p) \\
\sigma_{C E P}(p p \rightarrow p+J / \Psi J / \Psi+p) \\
\sigma_{D D}(p p \rightarrow p+\Upsilon \Upsilon+p) \\
\sigma_{C E P}(p p \rightarrow p+\Upsilon \Upsilon+p)\end{array}$ & $\begin{array}{c}11.4 \mathrm{pb} \\
439.2 \mathrm{pb} \\
4.7 \times 10^{-2} \mathrm{pb} \\
2.4 \times 10^{-2} \mathrm{pb}\end{array}$ & $\begin{array}{c}4.0 \mathrm{pb} \\
222.3 \mathrm{pb} \\
2.0 \times 10^{-2} \mathrm{pb} \\
1.4 \times 10^{-2} \mathrm{pb}\end{array}$ & $\begin{array}{c}2.0 \mathrm{pb} \\
90.4 \mathrm{pb} \\
8.8 \times 10^{-3} \mathrm{pb} \\
5.1 \times 10^{-3} \mathrm{pb}\end{array}$ \\
\hline
\end{tabular}

For the FCC energy, our results indicate that the contribution of the diffractive and central exclusive mechanisms will be similar.

In Table II, we present our predictions for the cross sections considering $p p$ collisions for the center-of-mass energies of the LHC, HE-LHC, and FCC, and different rapidity ranges. For the HE-LHC energy, we assume that $\left\langle S^{2}\right\rangle=0.015$. We predict cross sections of order of $\mathrm{pb}(\mathrm{fb})$ in the case of the double $J / \Psi(\Upsilon)$ production, which increases with the energy and are smaller in the forward rapidity range. For the central exclusive processes, the increase is steeper, which is expected since the cross section is proportional to the fourth power of the conventional gluon distribution while in the DD case the cross section is proportional to the square of the diffractive gluon distribution. In agreement with the results presented in Fig. 3, we have that double $J / \Psi$ production is dominated by the central exclusive production. On the other hand, for the double $\Upsilon$ production at the LHC, the DD process dominates. For larger energies, the contribution of the double diffractive and central exclusive processes becomes similar.

Finally, in order to estimate the dependence of our results on the parametrization used as input in the calculations, we have calculated the central exclusive and double diffractive processes considering two other parton distribution functions (PDFs). In the exclusive case, we have considered the CT14 parametrization [69] for the inclusive gluon distribution. For the double diffractive production, we have estimated the rapidity distributions using the Fit B for the diffractive parton distributions proposed in Ref. [32]. The results are presented in Fig. 4 for $p p$ collisions at the FCC energies, where the difference between the predictions is larger. For the central exclusive production, we have that CT14 parametrization implies smaller values for the
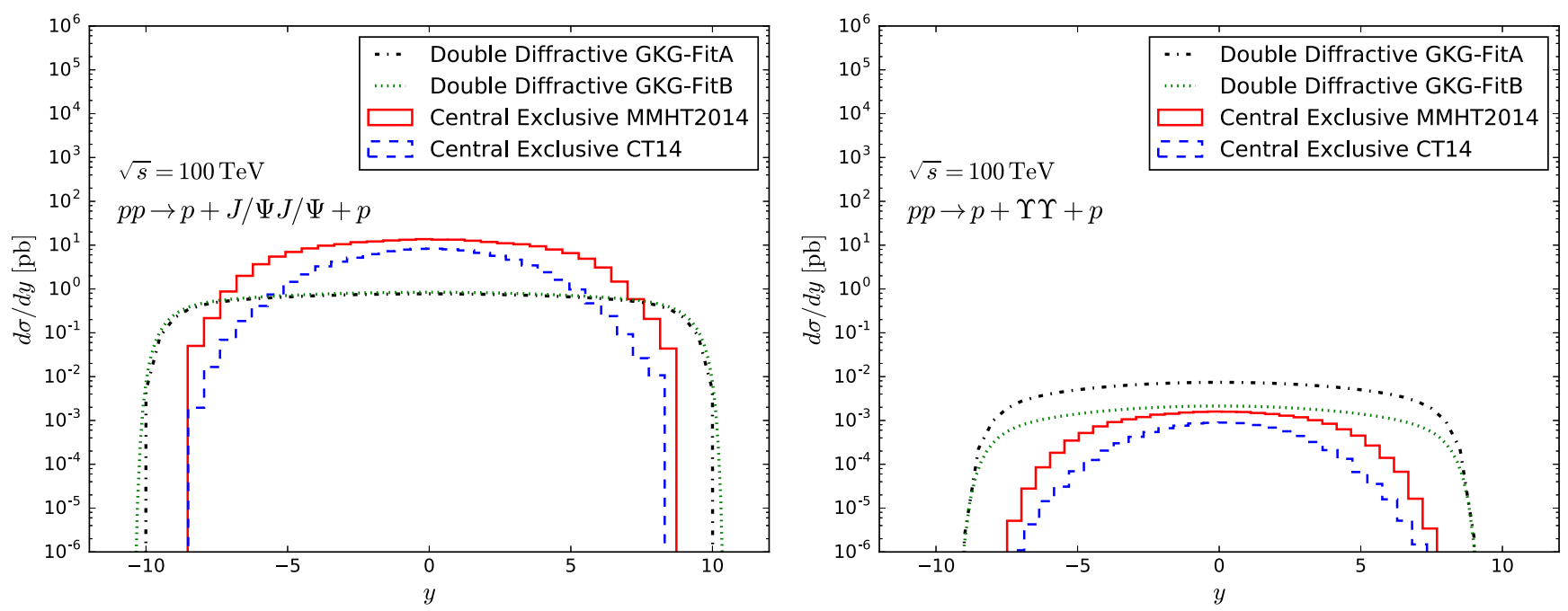

FIG. 4. Rapidity distributions for the diffractive and central exclusive double $J / \Psi$ (left panel) and double $\Upsilon$ (right panel) production in $p p$ collisions at the FCC considering different parametrizations for the gluon distribution function. 
rapidity distributions. We have verified that the total cross sections for the double $J / \Psi(\Upsilon)$ production are reduced by a factor $\approx 4(2)$. Such large dependence on the PDF used in the calculation is expected since the central exclusive cross section is $\propto(x g)^{4}$ and the MMHT and CT14 parametrizations are largely distinct at small $x$, which is the kinematical region probed at the FCC energy. This theoretical uncertainty is similar to that associated to the modeling of the soft survival factor, estimated in Table I. An alternative to improve our understanding of the central exclusive production and reduce the uncertainty in the predictions is the measurement of both final states $(J / \Psi J / \Psi$ and $\Upsilon \Upsilon)$, since they are differently affected by the soft survival effects and by the PDF used in the calculations. For the double diffractive production, we have that the FitA and FitB predictions are similar for the double $J / \Psi$ and differ for double $\Upsilon$, with the FitB implying smaller values for the rapidity distribution and a reduction by a factor 1.8 of the total cross section. Such result indicates that the measurement of the $J / \Psi J / \Psi$ final state can be useful to constrain the soft survival effects in the double diffractive production.

\section{SUMMARY}

In this paper, we have investigated the quarkonium-pair production in double diffractive and central exclusive processes considering $p p$ collisions at the LHC,
HE-LHC, and FCC energies. For the treatment of the double diffractive production, we have used the NRQCD factorization formalism for the quarkonium production and the resolved Pomeron model to describe the diffractive processes. On the other hand, in the case of central exclusive processes, we have considered the Durham model to describe the interaction. We estimated the rapidity and transverse momentum dependencies of the cross sections for the $J / \Psi J / \Psi$ and $\Upsilon \Upsilon$ production and presented predictions considering the kinematical rapidity ranges probed by central and forward detectors. The absorptive corrections have been included in our calculations assuming a simplistic model to treat the soft rescattering corrections. Our results demonstrated that the contribution of the central exclusive (double diffractive) processes can be separated selecting events where the transverse momentum of the pair is small (large). Our results indicate that the study of the quarkonium-pair production can be useful to test the underlying assumptions present in the description of the double diffractive and central exclusive processes.

\section{ACKNOWLEDGMENTS}

V. P. G. acknowledge useful discussions with M. Rangel and R. McNulty. This work was partially financed by the Brazilian funding agencies CNPq, CAPES, FAPERGS, and INCT-FNA (Process No. 464898/2014-5).
[1] V. G. Kartvelishvili and S. M. Esakiya, Yad. Fiz. 38, 722 (1983).

[2] B. Humpert and P. Mery, Z. Phys. C 20, 83 (1983).

[3] R. Vogt and S. J. Brodsky, Phys. Lett. B 349, 569 (1995).

[4] C. F. Qiao, Phys. Rev. D 66, 057504 (2002).

[5] R. Li, Y. J. Zhang, and K. T. Chao, Phys. Rev. D 80, 014020 (2009).

[6] C. F. Qiao, L. P. Sun, and P. Sun, J. Phys. G 37, 075019 (2010).

[7] P. Ko, J. Lee, and C. Yu, J. High Energy Phys. 01 (2011) 070 .

[8] A. V. Berezhnoy, A. K. Likhoded, A. V. Luchinsky, and A. A. Novoselov, Phys. Rev. D 84, 094023 (2011).

[9] A. P. Martynenko and A. M. Trunin, Phys. Rev. D 86, 094003 (2012).

[10] J. P. Lansberg and H. S. Shao, Phys. Rev. Lett. 111, 122001 (2013).

[11] Y. J. Li, G. Z. Xu, K. Y. Liu, and Y. J. Zhang, J. High Energy Phys. 07 (2013) 051.

[12] J. P. Lansberg and H. S. Shao, Nucl. Phys. B900, 273 (2015).

[13] J. P. Lansberg and H.S. Shao, Phys. Lett. B 751, 479 (2015).

[14] L. P. Sun, H. Han, and K. T. Chao, Phys. Rev. D 94, 074033 (2016).
[15] J. P. Lansberg, C. Pisano, F. Scarpa, and M. Schlegel, Phys. Lett. B 784, 217 (2018); 791, 420(E) (2019).

[16] C. H. Kom, A. Kulesza, and W. J. Stirling, Phys. Rev. Lett. 107, 082002 (2011).

[17] S. P. Baranov, A. M. Snigirev, and N. P. Zotov, Phys. Lett. B 705, 116 (2011).

[18] S. P. Baranov, A. M. Snigirev, N. P. Zotov, A. Szczurek, and W. Schafer, Phys. Rev. D 87, 034035 (2013).

[19] D. d'Enterria and A. M. Snigirev, Phys. Lett. B 727, 157 (2013); Nucl. Phys. A931, 303 (2014).

[20] J. R. Gaunt, R. Maciula, and A. Szczurek, Phys. Rev. D 90, 054017 (2014).

[21] C. Borschensky and A. Kulesza, Phys. Rev. D 95, 034029 (2017).

[22] A. V. Berezhnoy, A. K. Likhoded, and A. A. Novoselov, Phys. Rev. D 87, 054023 (2013).

[23] V. M. Abazov et al. (D0 Collaboration), Phys. Rev. D 90, 111101 (2014).

[24] M. Aaboud et al. (ATLAS Collaboration), Eur. Phys. J. C 77, 76 (2017).

[25] V. Khachatryan et al. (CMS Collaboration), J. High Energy Phys. 09 (2014) 094.

[26] R. Aaij et al. (LHCb Collaboration), Phys. Lett. B 707, 52 (2012); J. High Energy Phys. 06 (2017) 047; 10 (2017) 068. 
[27] R. Aaij et al. (LHCb Collaboration), J. Phys. G 41, 055002 (2014).

[28] K. Akiba et al. (LHC Forward Physics Working Group), J. Phys. G 43, 110201 (2016).

[29] C. Brenner Mariotto and V. P. Goncalves, Phys. Rev. D 91, 114002 (2015).

[30] C. Brenner Mariotto, V. P. Goncalves, and R. Palota da Silva, Phys. Rev. D 98, 014028 (2018).

[31] L. A. Harland-Lang, V. A. Khoze, and M. G. Ryskin, J. Phys. G 42, 055001 (2015).

[32] M. Goharipour, H. Khanpour, and V. Guzey, Eur. Phys. J. C 78, 309 (2018).

[33] A. Abada et al. (FCC Collaboration), Eur. Phys. J. Spec. Top. 228, 755 (2019).

[34] CMS and TOTEM Collaborations, CMS-TOTEM Precision Proton Spectrometer Technical Design Report, http://cds .cern.ch/record/1753795; M. Tasevsky (ATLAS Collaboration), AIP Conf. Proc. 1654, 090001 (2015).

[35] G. Ingelman and P. E. Schlein, Phys. Lett. B 152, 256 (1985).

[36] V. A. Khoze, A. D. Martin, and M. G. Ryskin, Eur. Phys. J. C 23, 311 (2002); 24, 581 (2002).

[37] G. T. Bodwin, E. Braaten, and G. P. Lepage, Phys. Rev. D 51, 1125 (1995); 55, 5853(E) (1997).

[38] E. Braaten, B. A. Kniehl, and J. Lee, Phys. Rev. D 62 , 094005 (2000).

[39] M. Kramer, Prog. Part. Nucl. Phys. 47, 141 (2001).

[40] E. J. Eichten and C. Quigg, Phys. Rev. D 52, 1726 (1995).

[41] C. Patrignani et al. (Particle Data Group), Chin. Phys. C 40, 100001 (2016).

[42] A. Aktas et al. (H1 Collaboration), Eur. Phys. J. C 48, 715 (2006).

[43] M. G. Ryskin, A. D. Martin, V. A. Khoze, and A. G. Shuvaev, J. Phys. G 36, 093001 (2009); V. A. Khoze, A. D. Martin, and M. G. Ryskin, J. Phys. G 45, 053002 (2018).

[44] G. P. Lepage and S. J. Brodsky, Phys. Rev. D 22, 2157 (1980).

[45] V. L. Chernyak and A. R. Zhitnitsky, Phys. Rep. 112, 173 (1984).

[46] L. A. Harland-Lang, V. A. Khoze, and M. G. Ryskin, Eur. Phys. J. C 79, 39 (2019).

[47] L. A. Harland-Lang, A. D. Martin, P. Motylinski, and R. S. Thorne, Eur. Phys. J. C 75, 204 (2015).

[48] J. D. Bjorken, Phys. Rev. D 47, 101 (1993).
[49] J. C. Collins, Phys. Rev. D 57, 3051 (1998); 61, 019902(E) (1999).

[50] C. O. Rasmussen and T. Sjostrand, J. High Energy Phys. 02 (2016) 142.

[51] S. Ostapchenko and M. Bleicher, Eur. Phys. J. C 78, 67 (2018).

[52] S. Gieseke, F. Loshaj, and P. Kirchgaeber, Eur. Phys. J. C 77, 156 (2017).

[53] V. A. Khoze, A. D. Martin, and M. G. Ryskin, Eur. Phys. J. C 73, 2503 (2013).

[54] V. P. Goncalves, R. P. da Silva, and P. V. R. G. Silva, Phys. Rev. D 100, 014019 (2019).

[55] V. P. Goncalves, C. Potterat, and M. S. Rangel, Phys. Rev. D 93, 034038 (2016).

[56] M. V. T. Machado, Phys. Rev. D 76, 054006 (2007); M. B. Gay Ducati, M. M. Machado, and M. V. T. Machado, Phys. Rev. D 81, 054034 (2010); M. B. Gay Ducati, M. M. Machado, and M. V.T. Machado, Phys. Rev. C 83, 014903 (2011).

[57] M. Luszczak, R. Maciula, and A. Szczurek, Phys. Rev. D 84, 114018 (2011).

[58] M. Luszczak, R. Maciula, and A. Szczurek, Phys. Rev. D 91, 054024 (2015).

[59] C. Brenner Mariotto and V. P. Goncalves, Phys. Rev. D 88, 074023 (2013).

[60] A. K. Kohara and C. Marquet, Phys. Lett. B 757, 393 (2016).

[61] E. Basso, V. P. Goncalves, and M. S. Rangel, Eur. Phys. J. C 76, 689 (2016).

[62] E. Basso, V. P. Goncalves, A. K. Kohara, and M. S. Rangel, Eur. Phys. J. C 77, 600 (2017).

[63] V. P. Goncalves, M. M. Jaime, D. E. Martins, and M. S. Rangel, Phys. Rev. D 97, 074024 (2018).

[64] E. A. F. Basso, V. P. Goncalves, D. E. Martins, and M. S. Rangel, Phys. Rev. D 99, 034017 (2019).

[65] V. A. Khoze, A. D. Martin, and M. G. Ryskin, Eur. Phys. J. C 18, 167 (2000).

[66] V. A. Khoze, A. D. Martin, and M. G. Ryskin, Int. J. Mod. Phys. A 30, 1542004 (2015).

[67] E. Gotsman, E. Levin, and U. Maor, Int. J. Mod. Phys. A 30, 1542005 (2015).

[68] A. Abada et al. (FCC Collaboration), Eur. Phys. J. Spec. Top. 228, 1109 (2019).

[69] S. Dulat, T.-J. Hou, J. Gao, M. Guzzi, J. Huston, P. Nadolsky, J. Pumplin, C. Schmidt, D. Stump, and C.-P. Yuan, Phys. Rev. D 93, 033006 (2016). 